\title{
Non-uniform structures in low-density nuclear matter and neutron star crust
}

\author{
Minoru Okamoto $^{* a b}$, Toshiki Maruyama ${ }^{b}$, Kazuhiro Yabana $^{c a}$ and Toshitaka Tatsumi ${ }^{d}$ \\ ${ }^{a}$ Graduate School of Pure and Applied Science, University of Tsukuba \\ Tennoudai 1-1-1, Tsukuba, Ibaraki 305-8571, Japan \\ ${ }^{b}$ Advanced Science Research Center, Japan Atomic Energy Agency \\ Shirakata Shirane 2-4, Tokai, Ibaraki 319-1195, Japan \\ ${ }^{c}$ Center of Computational Sciences, University of Tsukuba \\ Tennoudai 1-1-1, Tsukuba, Ibaraki 305-8571, Japan \\ ${ }^{d}$ Department of Physics, Kyoto University \\ Kyoto 606-8502, Japan \\ E-mail: okamoto@nucl.ph.tsukuba.ac.jp maruyama.toshiki@jaea.go.jp \\ yabana@nucl.ph.tsukuba.ac.jp|tatsumi@ruby.scphys.kyoto-u.ac.jp
}

\begin{abstract}
In neutron star crust, non-uniform structure of nuclear matter are expected which is called "pasta" structure. From the recent studies of magnetar giant flares, these structures might be related to some observables and physical quantity of neutron star crust. In the perspective of the calculation for above quantities, we numerically explore the pasta structures and properties of low-density catalyzed nuclear matter based on the relativistic mean-fields model and Thomas-Fermi approximation with a fully three-dimensional geometry. We observe two type of conventional pasta structures. We also discuss the crystalline structure of "pasta".
\end{abstract}

XII International Symposium on Nuclei in the Cosmos,

August 5-12, 2012

Cairns, Australia

\footnotetext{
* Speaker.
} 
Recently, in the tails of magnetar giant flares, quasi-periodic oscillations (QPOs) have been observed [1]. These flares are energetic $\gamma$-ray bursts from strongly magnetized neutron stars. The most comprehensible unerstanding of the QPOs is the shear oscillations on neutron star crust. Based on this interpretation, detailed information on neutron star crust can be obtained from the QPOs. The frequencies of shear oscillations depend on shear moduli which is characterized by Coulomb solid of nuclei. Ogata et al. have calculated shear moduli using Molecular dynamics simulations [2]. But, in this calculation, some important effects are absent, such as Coulomb screening, finite size effects of nuclei, superfluidity of dripped neutron and so on. Considering the realistic situation, these components should be taken into account in the precise calculation of shear modulus.

Besides, in the crust, not only spherical nuclei but also other shapes of nuclear matter are expected [3]. Toward the inside of neutron star crust, the shape of constituent nuclei is expected to change from spherical droplet to cylindrical rod, slab, cylindrical tube, spherical bubble, and to uniform. These structures are figuratively called as "nuclear pasta".

The density-dependence of the species and the sizes of pasta are determined by minimizing the total energy density, i.e. the sum of the bulk, the surface, and the Coulomb energy densities. From thermodynamics of view, nuclear matter at sub-saturation density consists of a dilute gas phase and a dense liquid phase in chemical equilibrium which determines particle densities in both phases. Thus the bulk energy density is independent of the structure. Once the averaged density is given, the volume fraction is determined. However, the shape and the size of the structure are determined by the balance between the surface and the Coulomb energy densities.

In many studies on nuclear pasta, the geometrical symmetry of the structure is assumed, i.e. the Wigner-Seitz (WS) approximation is employed [4, 5]. In this approximation, all the physical quantities of matter are represented by those of a single WS cell. Furthermore, the calculation can be reduced by one-dimensional problem owing to the geometrical symmetry, which drastically saves the computational cost. On the other hand, there are several works by mean-fields calculation without any assumption on the geometry 60 . These studies have used periodic boundary condition on a small cubic cell, which included one period of structures. Although they got the same results, i.e. typical pasta structures, it is hard to discuss further information like crystalline structure of "pasta" and mechanical properties.

For detailed studies on the properties of neutron star crust, it has been desired to perform three-dimensional calculations in a periodic cubic cell with sufficiently large sizes. In this article, we explore pasta structures and properties of low-density catalyzed nuclear matter. Appearance of pasta structures and others complex structures, crystalline structure of droplets will be discussed. Estimation of shear modulus in neutron star crust matter with pasta structures will be studied elsewhere.

To describe the baryon interaction, we employ a relativistic mean-field (RMF) model with the Thomas-Fermi approximation [8]. It deals with fields of mesons and baryons by a Lagrangian introduced in a Lorentz-invariant way. From the variational principle, we get the coupled equations of motion for the mean-fields and the Coulomb potential as

$$
-\nabla^{2} \sigma(\mathbf{r})+m_{\sigma}^{2} \sigma(\mathbf{r})=g_{\sigma N}\left(\rho_{p}^{s}(\mathbf{r})+\rho_{n}^{s}(\mathbf{r})\right)-\frac{d U}{d \sigma}
$$




$$
\begin{aligned}
-\nabla^{2} \omega_{0}(\mathbf{r})+m_{\omega}^{2} \omega_{0}(\mathbf{r}) & =g_{\omega N}\left(\rho_{p}(\mathbf{r})+\rho_{n}(\mathbf{r})\right) \\
-\nabla^{2} R_{0}(\mathbf{r})+m_{\rho}^{2} R_{0}(\mathbf{r}) & =g_{\rho N}\left(\rho_{p}(\mathbf{r})-\rho_{n}(\mathbf{r})\right) \\
\nabla^{2} V_{\text {Coul }}(\mathbf{r}) & =e^{2}\left(\rho_{p}(\mathbf{r})+\rho_{e}(\mathbf{r})\right)
\end{aligned}
$$

where $\rho_{i}^{s}(\mathbf{r})=\left\langle\bar{\psi}_{i}(\mathbf{r}) \psi_{i}(\mathbf{r})\right\rangle, i=p, n$ is the nucleon scalar density and the non-linear potential for the scalar field $U(\sigma)=\frac{1}{3} b m_{N}\left(g_{\sigma N} \sigma\right)^{3}-\frac{1}{4} c\left(g_{\sigma N} \sigma\right)^{4}$. Equations of motion for fermions simply yield the standard relations between the densities and chemical potentials,

$$
\begin{aligned}
\mu_{n} & =\mu_{B}=\sqrt{k_{\mathrm{F}, n}(\mathbf{r})^{2}+m_{N}^{*}(\mathbf{r})^{2}}+g_{\omega N} \omega_{0}(\mathbf{r})-g_{\rho N} R_{0}(\mathbf{r}) \\
\mu_{p} & =\mu_{B}-\mu_{e}=\sqrt{k_{\mathrm{F}, p}(\mathbf{r})^{2}+m_{N}^{*}(\mathbf{r})^{2}}+g_{\omega N} \omega_{0}(\mathbf{r})+g_{\rho N} R_{0}(\mathbf{r})-V_{\mathrm{Coul}}(\mathbf{r}) \\
\rho_{e}(\mathbf{r}) & =-\left(\mu_{e}-V_{\mathrm{Coul}}(\mathbf{r})\right)^{3} / 3 \pi^{2}
\end{aligned}
$$

where $m_{N}^{*}(\mathbf{r})=m_{N}-g_{\sigma N} \sigma(\mathbf{r})$ represents an effective mass of nucleon. We assumed that the system is in chemical equilibrium among nucleons and electrons and introduced the baryon-number chemical potential $\mu_{B}$ and the electron-number chemical potential $\mu_{e}$. It is rather simple for numerical calculations, but realistic enough to reproduce the bulk properties of nuclear matter and finite nuclei. We use the same parameters as in Ref. [8] so as to compare the EOS and structural changes of pasta with or without WS approximation. In these parameters, we can represent the properties of uniform nuclear matter showing in Table1

\begin{tabular}{ccccc}
\hline$\rho_{0}\left[\mathrm{fm}^{-3}\right]$ & $\varepsilon_{0}[\mathrm{MeV}]$ & $K[\mathrm{MeV}]$ & $S_{0}[\mathrm{MeV}]$ & $L[\mathrm{MeV}]$ \\
\hline 0.153 & -16.4 & 240 & 33.4 & 84 \\
\hline
\end{tabular}

Table 1: EOS of uniform nuclear matter.

To numerically simulate infinite matter, we use a cubic cell with periodic boundary conditions. We divide the cell into three-dimensional grids. The desirable cell size should be as large as to include several periods of pasta structures, and the desirable grid width should be as small as to describe the detailed density distribution at nuclear surface. If the cell size is small that includes only one or two periods of structure, its shape should be affected by the boundary condition and the appearance of some structures is implicitly suppressed.

Giving average baryon density $\rho_{B}$, initial density distributions of fermions are randomly prepared on each grid points. Then proper density distributions and meson fields are searched for. Detailed calculation method is reported in Ref. [9].

Shown in Fig. 1 are the proton density distributions in cold catalyzed nuclear matter. We observe body-centred cubic (bcc) on droplets,face-centred cubic (fcc) on droplets, honeycomb lattice of rod, depending on the density. In Fig 2 the density profiles of fermions in bcc and fcc lattice of droplets and Coulomb potentials are plotted along a line which crosses through the droplets. For $\rho_{B} \approx 0.01 \mathrm{fm}^{-3}$ and $\rho_{B} \approx 0.03 \mathrm{fm}^{-3}$, the effect of Coulomb repulsion can be seen where the proton density is highest near the surface, while the neutron density distribution in the droplet is flat. We have observed only those three structures (a), (b), (c) in Fig. 1. This result is consistent with the previous study about the relation between the density region of pasta structure and the differential coefficient of symmetry energy $L[10]$. The larger $L$ yields, the narrower pasta region is. In our model and parameters, differential coefficient of symmetry energy is about $80 \mathrm{MeV}$, which is 


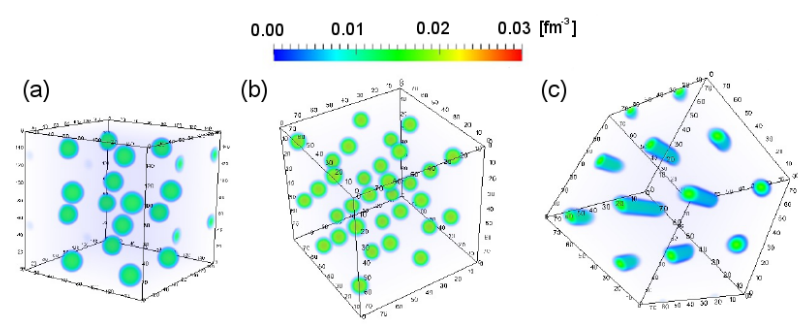

Figure 1: Proton density distribution are shwon; droplet (bcc) at $\rho_{B}=0.01 \mathrm{fm}^{-3}$, droplet (fcc) at $\rho_{B}=0.03 \mathrm{fm}^{-3}$, $\operatorname{rod}\left(\right.$ honeycomb) at $\rho_{B}=0.056 \mathrm{fm}^{-3}$
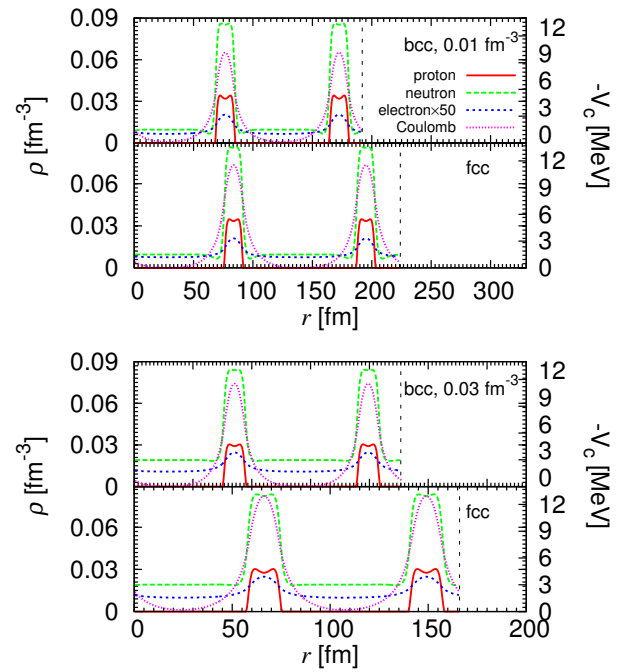

Figure 2: (color online) Density distribution of proton, neutron, and electron and Coulomb potential in fcc and bcc. Upper part shows the case of $\rho_{B}=0.01 \mathrm{fm}^{-3}$ and $\rho_{B}=0.03 \mathrm{fm}^{-3}$ in lower panel. Red lines indicate proton, green neutron, blue electron (50 times) density distribution, pink Coulomb potential and black dashed lines are the lattice constant

close to critical value $\approx 90 \mathrm{MeV}$. In the previous calculation using WS approximation, only the droplet structure appeared as ground state. But in our three-dimensional calculation using same RMF framework, rod structure also appear.

We show the density dependence of the total energy, Coulomb energy and proton fraction in Fig. 3 To see the difference between droplet in bcc and fcc lattice, we plot the density dependence of the size of droplet, lattice constant, volume fraction, proton number of each droplet and proton fraction in Fig. 4 Here, $R_{d}$ and $R_{\text {cell }}$ are defined as follows, $\frac{V}{N_{d}}=\frac{4 \pi}{3} R_{\text {cell }}^{3}, R_{d}=R_{\text {cell }}\left(\frac{\left\langle\rho_{p}\right\rangle^{2}}{\left\langle\rho_{p}^{2}\right\rangle}\right)^{1 / 3}$ where $V$ denotes the cell volume, $N_{d}$ the number of droplets in the cell, and the bracket $\langle\ldots\rangle$ the average over the cell volume.
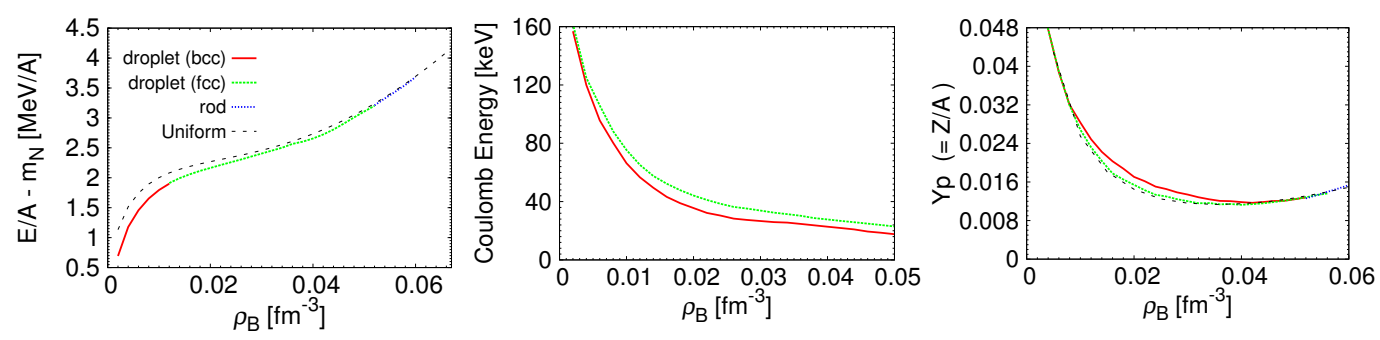

Figure 3: (color online) From the left, total energy, Coulomb energy, and proton fraction. Red lines indicate droplet (fcc),green droplet (bcc), blue rod, respectively. Dotted lines are the case of single phase.

The density dependence of ground state energy is shown in the left panel of Fig. 3 We can see that grand state configuration changes depending on the density. In the lower density regions, bcc of droplets lattice appear. Around $\rho_{B} \approx 0.01 \mathrm{fm}^{-3}$, lattice structure changes from bcc to fcc lattice. 

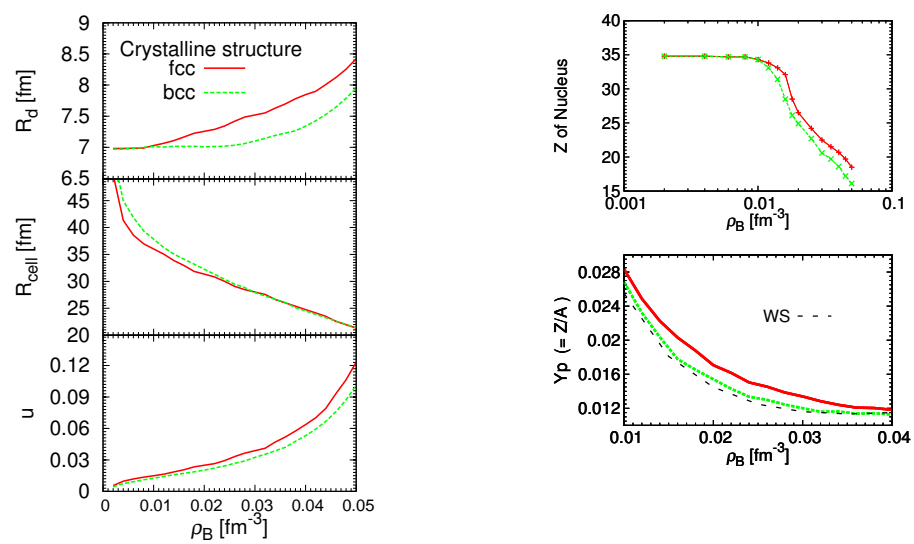

Figure 4: (color online) In the left, the density dependence of radii of nuclei, and lattice constants and proton number of nuclei and proton fraction in the right panel. The lines in each panel are represented as red line indicate fcc, green bcc.

And drastic change occur around $\rho_{B} \approx 0.052 \mathrm{fm}^{-3}$ : from fcc lattice of droplets to honeycomb lattice of rods. It is hard to recognize the total energy difference between bcc and fcc lattices of droplets. That of proton fraction at $\rho_{B}<0.01 \mathrm{fm}^{-3}$ in the right panel of Fig. 3 is also hard to distinguish. However, there are significant differences in the proton fraction at $\rho_{B}>0.01 \mathrm{fm}^{-3}$ and Coulomb energy (middle panel of Fig 3 .

As we have seen, one of new results appears in the crystalline structure of droplets. In our calculation, it emerges as a fcc lattice near the transition density from droplet to rod, while it has been regarded to take a bcc lattice in the previous studies [11]. Crystalline structures in the bcc and fcc lattice give rise to a subtle difference of the proton fraction and size of droplets. In Ref. [11], it is reported that the bcc crystalline structure of droplets is realized in the ground state at low densities, using compressible liquid drop model (CLDM). In this calculation, same radii of droplet and proton fraction are assumed when the total energies of different crystalline structure are compared. Then, the energy difference between bcc and fcc lattice comes only from the Coulomb energy. In low-density limit, same assumptions are realized in our calculation: Almost same radius and density distribution of droplet, proton fraction and proton number in nuclei for both crystalline structure (see the right panel of Fig. 4 at $\rho_{B}<0.01 \mathrm{fm}^{-3}$ ). The difference between bcc and fcc lattice structure can be seen only in the Coulomb energy. Coulomb potential of fcc lattice is little higher than that of bcc. However, near the transition density from droplet to rod, the radii of droplet and proton fraction are not same between bcc and fcc lattice even if their baryon densities are the same (see Fig. 4 at $\rho_{B} \approx 0.02 \mathrm{fm}^{-3}$ ). The size of droplet and proton fraction in fcc lattice is 7.54 $\mathrm{fm}$ and 0.0172 respectively, meanwhile those of bcc is $7.01 \mathrm{fm}$ and 0.0158 . Because the size of droplet and proton fraction are different, the Coulomb energy is no more the criterion of ground state. We should take account the size of droplet and proton fraction for searching the ground state in self-consistent way. Roughly, the larger the radius of droplet is, the smaller the surface energy is. While the Coulomb energy of fcc lattice is larger than that of bcc in all the region of droplet, the total energy of fcc lattice is less than that of bcc by the gain of surface energy.

The maximum size of droplet can be estimated by the Bohr-Wheeler condition as $\mathrm{E}_{\mathrm{Coul}}^{(0)}>$ 
$2 \mathrm{E}_{\text {surf }}$ [12], the optimum size condition $\mathrm{E}_{\text {surf }}=2 \mathrm{E}_{\text {Coul }}$ where $\mathrm{E}_{\text {Coul }}^{(0)}$ is the Coulomb energy of a isolated nucleus and the Coulomb energy of a nucleus in matter as $\mathrm{E}_{\mathrm{Coul}} \approx \mathrm{E}_{\mathrm{Coul}}^{(0)}\left(1-3 \mathrm{u}^{1 / 3} / 2\right)$. From these equations, the appearance of non-spherical nucleus in nuclear matter has been expected for the volume fraction $u>0.125$. However, in our calculation, the structural change from droplet to rod occurs around $u \approx 0.1$ (see the value at $\rho_{B} \approx 0.05 \mathrm{fm}^{-3}$ in Fig. (4). This means that considering the non-uniformity of electron is worthwhile for "pasta" structures, because the relation between the Coulomb energies of a cell and that of a nucleus has been derived by using a uniform background electrons and uniform baryon density in a nucleus. The effect of the screening by charged particles, which is naturally included in our calculation, may be one of the origins of this difference. To get the final conclusion of this issue, we should perform another calculation with uniformly distributed electrons and confirm the effects of non-uniformity of electron. Also the difference of the droplet surface may be the origin, since they used the CLDM with a sharp surface, while our droplets have a diffuse surface. The same discussion may occur for the Quantum molecular dynamics calculation [13]. They got the droplet of bcc lattice without any assumption on geometry with uniform electron background. This difference of the crystalline structure between these calculations and the present calculation remains as a future problem.

We have numerically explored non-uniform structures and properties of low-density cold catalyzed nuclear matter using the RMF model and the Thomas-Fermi approximation. Without any assumption on the geometry, we have carried out fully three-dimensional calculations in large cubic cells with periodic boundary conditions. With increase of density, which ranges from well below to half of the normal nuclear density, we have observed that the ground state of matter shows two types of pasta structures. As for the crystalline structures of droplets, near the transition density from droplet to rod, fcc lattice has been more suitable than bcc, which is different from the previous studies.

\section{References}

[1] Tod E. Strohmayer and Anna L. Watts, ApJ. 632 L 111 (2005).

[2] S. Ogata and S. Ichimaru, Phys. Rev. A 424867 (1990).

[3] P. Haensel, A. Y. Pothekhin, D. G. Yakovlev, Neutron Stars 1 (Springer, 2007).

[4] G. D. Ravenhall, J. C. Pethick, R. J. Wilson, Phys. Rev. Lett. 50, 2066 (1983).

[5] M. Hashimoto, H. Seki, M. Yamada, Prog. Theor. Phys. 71, 320 (1984).

[6] R. D. Williams, S. E. Koonin, Nucl. Phys. A 435, 844 (1985).

[7] W. G. Newton, J. R. Stone, Phys. Rev. C 79, 055801 (2009).

[8] T. Maruyama, T. Tatsumi, D. N. Voskresensky, T. Tanigawa, S. Chiba, Phys. Rev. C 72, 015802 (2005).

[9] M. Okamoto, T. Maruyama, K. Yabana, T. Tatsumi, Phys. Lett. B 713284 (2012)

[10] K. Oyamatsu, K. Iida, Phys. Rev. C 75, 015801 (2007)

[11] K. Oyamatsu, M. Hashimoto, M. Yamada, Prog. Theor. Phys. 72, 373 (1984).

[12] M. A. Preston, R. Bhaduri, Structure of the Nucleus (Addison-Wesley Publishing Company, 1975).

[13] G. Watanabe, K. Sato, K. Yasuoka, T. Ebisuzaki, Phys. Rev. C 68, 035806 (2003). 